\title{
Téoros
}

Revue de recherche en tourisme

\section{Le transport aérien et sa problématique dans les pays en développement}

\section{Gabriel Wackermann}

Volume 13, numéro 2, été 1994

Regards sur le tourisme dans les pays en développement

URI : https://id.erudit.org/iderudit/1077759ar

DOI : https://doi.org/10.7202/1077759ar

Aller au sommaire du numéro

Éditeur(s)

Université du Québec à Montréal

ISSN

0712-8657 (imprimé)

1923-2705 (numérique)

Découvrir la revue

Citer cet article

Wackermann, G. (1994). Le transport aérien et sa problématique dans les pays en développement. Téoros, 13(2), 21-24. https://doi.org/10.7202/1077759ar d'utilisation que vous pouvez consulter en ligne.

https://apropos.erudit.org/fr/usagers/politique-dutilisation/ 


\section{Le transport aérien et sa problématique dans les pays en développement}

\section{Gabriel Wackermann"}

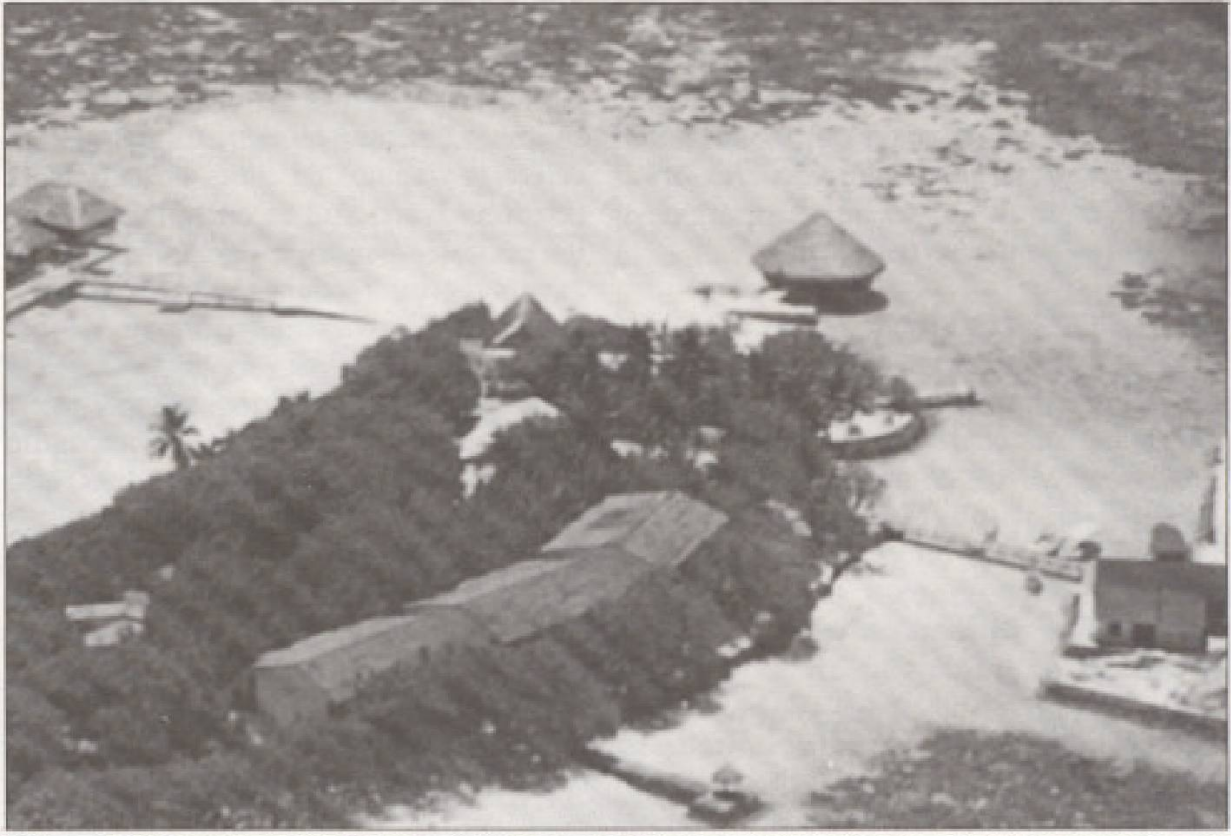

Grîce au développement du transport aérien, même les îles les plus éloignées sont devenues accessibles aux touristes.

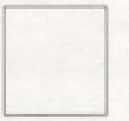

Le transport aérien dans son ensemble a commencé par devenir un outil de développement au service des pays du TiersMonde au lendemain de la Seconde Guerre mondiale, dès lors que les hostilités avaient suscité desenjeux tels que les Alliés devaient faire appel à toutes les matières premières età toutes lespositions stratégiques dontils pouvaient disposer sur leglobe. La rapidité relative des acheminements et les contacts directs avec l'intérieur des continents suscités par la voie aérienne ont permis de bouleverser fondamentalementlesrapports entre les pays industrialisés et les pays en développernent d'une part, entre ces derniers d'autre part. Certes le transport maritime demeure le moyen de circulation essentiel du fret. Mais dans le domaine concerné par la présente publication, le tourisme, l'avion avait beau jeu de remplacer le paquebot, à tel point qu'il fallut inventer ultérieurement le tourisme de croisière pour rendre à la mer en divertissements et découvertes ce quel'océan avait perdu en panache de longue durée. L'imagerie liéeà la chanson Quand le bateau arrivera à Tahiti est désormais reportée de fait sur l'atterrissage et l'accueil polynésien à l'aéroport de Papeete. Le déploiement touristiques'appuiede façon étroite sur des clientèles qui, pour des destinations lointaines ou des distances moyennes, surtout si celles-ci se heurtent à des parcours terrestres difficiles et fatigants, ont recours au vol. Dès la fin des années 1950 et surtout durant les années 1960, l'aménagement d'aéroports est devenu pour les porteurs de projets touristiques en pays en voie de développement un impératif premier, davantage encore qu'en pays avancé où des connexions et interconnexions terrestres à grande vitesse arrivent aux portes même d'imposants complexes de loisirs qui soustendent un tourisme commercial de grande envergure, tel Eurodisney, déjà implanté au voisinage de l'aéroville de Roissy, dont la gare de Marne-la-Vallée-Chessy, inaugurée en mai 1994, permet l'accès aux réseaux nord et sud-est du TGV par l'intermédiaire d'une ligne d'interconnexion contournant le centre de l'Ile-de-France.

\section{Le désenclavement}

Les pays en développement, même lorsqu'ils disposent d'une façade maritime, 
souffrent d'une réelle marginalisation inhérente à l'absence ou à la faiblesse des axes, chaînes et réseaux logistiques qui déterminent ailleurs les grands flux de biens et de personnes. Les pays littoraux sont toutefois avantagés par rapport aux Etats enclavés dans la mesure où ils associent transports maritimes et transports aériens, parfois dans une certaine proportion des segments de transports terrestres. Même dans ce cas la promotion de la voie aérienne futvivementencouragée et continueà faire l'objet de sollicitations multiples. Dès lors que le fret entre en jeu, le transport de voyageurs s'en trouve conforté.

Les pays du Tiers-Monde sans accès à la mer sont d'autant plus tributaires del'avion que celui-ci constitue leur seul moyen d'acoès international direct. Cettesituation fragilise cependant ce mode de transport qui ne permet pas aux aétoports concernés de s'intégrer à un concept global de la circulation s'appuyant sur des structures plurimodales d'acheminement qui confortent le tra fic et réduisent la fragilité de la rentabilité. Pourtant, en dépit de ce genre d'isolement de l'aéroport en pays peu développé, de surcroîtcoupé de la mer par un découpageterritorial dontladécolonisation a fréquemment amplifié les inconvénients, les liaisons aériennes sont devenues vitales pour ledevenir dessociétés démunies. Aussi les dirigeants de celles-ci ne furent-1ls pas trop regardants et ne le sont-ils toujours pas systématiquement lorsque le tourisme vint ou vient en quelque sorte comme renfort de plates-formes ayant beaucoup de malà se maintenir sans soutien matếriel de toute sorte.

Le désenclavement ne bénéficie pas seulement aux pays sans rivage maritime; il dépasse cette position stricto sensu chaque fois qu'un pays du Tiers-Monde parvient à șe rattacher aux réseaux mondiaux, aussi faiblement soit-il. Manaus e.a., en pleine forêt amazonienne, n'a guère pu bénéficier de l'immense bassin fluvial pour se connecter au littoral, ni de l'autoroute transamazonienne projetée mais jamais réalisée. Seul l'aéroport exploité par la firmeVarigen mêmetempsquelecomplexe hôtelicr de grand standing ont autorisé le désenclavement et l'ouverture touristique de la région alentour auxvisiteursnationaux ou internationaux. L'Océanie, le monde insulaire de l'Océan Indien ou méditerranéen rythment une part déterminante sinon la totalité de leurs activités selon les flux et reflux touristiques actionnés par les tours-opérateurs par le truchement des aéroports.

Quoique bon nombre d'aéroports de ce type soient davantageliés aux mouvements de charters, donc intermittents, qu'aux lignes régulières, déficitaires, ils induisent de l'activité, conduisant à de profondes mutations socioculturelles et environnementales. La politiquedes prix pratiquée par les compagnies de charters concurrençant fortement les lignes régulières accélère la fragilité de celles-ci dans leurs relations Nord-Sud et encourage même les pouvoirs publics à demander à leurs agents d'avoir recours aux charters pour leurs déplacements internationaux de service. C'est le cas des fonctionnaires du Burkina Faso depuis l'époque du président Sankara, qui a mis à profit la compagnie créée par Le Point pour se lancer dans cette pratique.

Les exigences de la Seconde Guerre mondiale ont accéléré le progrès technologique et les créations de pistes déclencheuses d'insertion internationale des pays sous-développés. L'émergence du tourisme commercial a donc été favorisée par les conflits militaires qui ont contribué à créer de façon inespérée les infrastructures de base pour un emol économique ultérieur. En Polynésie française par exemple, la marineetle génie des forces américaines stationnées dans le Pacifique ont construit en 1943 sur le Motu Mute la premièrepisted'aviation del'Océanie, celle de Bora-Bora, d'une longueur de 2000 mètres. Dèsaprèsleconflitfurentinaugurés des vols civils. De 1946 à 1961, les vols internationaux firent escale à Bora-Bora; pour rejoindre par la voie aérienne Tahiti, les passagers eurent recours à un hydravion. A partir de 1954, le Catalina de la Régie aérienne interinsulaire (RAI) assurait, de lagon à lagon, les liaisons entre les llesde la Société: Bora-Bora, Raiatea et Tahiti. En 1961 fut mis en service l'aéroport international de Tahiti-Faaa qui plaça BoraBoraà 45 minutesde Tahiti et achemina les touristes-bananes vers les îles, appelés ainsi à cause de leurs coups de soleil cuisants tirant la peau.

Plus lecadrephysiqueet socialétaitattractif, maisplusl'économiedemeurait faible, plus les promoteurs du tourisme exerçaient leur emprise sur les pays considérés. Il en est encore de même de nos jours. Evoquons à titred'illustration l'aménagement de la base aérienne sur l'Annapurna, à 3600 mètres d'altitude, sur le chemin du trek qui cerne ce massif. L'installation, achevée en 1994 , est motivée par l'explosion touristique: dès 1991 les seuls touristes français furent près de 30000 à effectuer le voyage au Népal. En dépit des difficultés liées au milieu physiqueetàl'armée deporteursnécessaires à l'acheminement du matériel, les travaux s'annoncent rentables financièrement; la main-d'oeuvre est bon marché, travaillant pour quelques centaines de roupies. $\mathrm{La}$ réalisation donne directement accès aux pistes de trek sans qu'il ne soit nécessaire de passer par Katmandou, la capitale, distante de quelques centaines de kilomètres. Le woyage en avion inclut le survol de l'Everest.

Le transport aérien offre ainsi des potentialités considérables. Il est à même de mettre le globe au service du tourisme commercial et de répandre l'idée qu'il participe à la libération de l'espace. Les moyens dont il dispose à présent sont tels qu'il s'insère aisément aux stratégies des groupes financiers qui se sont assurés la maîtrise du fonctionnement des mécanismes qui régissent l'économie mondiale. L'emprise exercée par les grandes firmes sur les pays en développement relève en premier lieu de l'aptitude de celles-ci à s'appuyer sur des technologies hautement performantes de l'aviation susceptibles de réduire les distances à un point tel qu'elles deviennent compatibles avec la durée somme toute limitée des congés et avec le pouvoir d'achat des catégories moyennes des populations de la Triade.

\section{Les effets structurants du transport aérien}

L'organisation du transport aérien s'inscrit dans une logique de marché qui en appelle à toutes les facettes de l'économie, en l'occurrence depuis la prise en compte des ressources touristiques d'un pays ou d'une région jusqu'a la mise en oeuvre de productions de biens et services nécessaires ou utiles à la bonne marche de l'offre touristique. Capitaux et flux financiers, savoir et savoir-faire, technologies et équipements divers font partie intégrante des apports dont l'avion constitue le véhicule; seuls les équipements lourdset les produits demasse sontacheminéspar bateau. Déjà letourisme en soi s'accompagne de chaînes hôttelières, de restauration, de points de vente variés. Intégré désormais à des structures logistiques dont se dotent toutes les firmes tant soit peu efficaces, il ne peut plus guère être 
traité comme un secteur d'activité à part. D'essence tertiaro-industriel, il s'inscrit dans le cadre de chaines logistiques dans lesquelles le transport n'apparaît plus principalement comme inducteur d'activités. Bien au contraire, le transport relève des impulsions en provenance des toursopérateurs et des groupes d'intérêt - financiers et autres - qui les sous-tendent. Insensiblement les pays en voie de développementattractifsen matière touristique ont été englobés dans les réseaux qui recomposent les chaînes pour être mieux à même de répondre à l'intérêt qui leur est témoigné.

Dans cette nouvelle configuration, les divers types de Tiers-Mondes figurent comme de réelles dépendances logistiques des centres d'impulsion de la Triade à partir desquels sont conçus, réalisés et gérés les productions de biens et services liés au tourisme. La part des flux immatériels augmente constamment par rapport aux transports qui concrétisent les déplacements physiques. La chaine logistique s'enrichit progressivement d'apports télématiquesquigarantissent unemeilleure information, une régularité et une sécurité prononcées, une organisation et un suivi plus efficaces des voyages. Les pays en développement parviennent souvent par le biais du tourisme à bénéficier des technologies informatisées qui conditionnent leurs relations avec les Erats tertiaro-industricls dans le cadre de la nouvelle organisation internationale du travail. Les compagnies aériennes s'inscrivent dans ce cadre et se situent dans une hiérarchie de pouvoirs en fonction de leur capacité à maîtriser et à conquérir des parts de marché, appuyées sur des ressorts financiers et bancaires, ainsi que sur des tours-opérateurs plus ou moins influents. A travers ces toiles d'araignées se façonne également une hiérarchie d'aéroports tributaires des bubs qui captent et répartissent les flux de voyageursen fonction des besoins exprimés par les bassins émetteurs, c'est-à-dire les pays à pouvoir d'achat relativement élevé, mais aussi en vertu de stratégies de marché exprimées en termes de profit et/ou de concurrence. Les grosses firmes privées surtout, les groupes qui demeurent encore publics dans une économie de plus en plus tournée vers la libéralisation des marchés, visent l'appui des collectivités territoriales et des gouvernements de la Triade, de même que celui des institutions et collectivités en place des pays d'accueil pour rentabiliser au maximum leurs investisse- ments dont le transport aérien représente tant l'outil de concrétisation finale des flux que le poste budgétaire qu'il convient de ramener au minimum incompressible, afin que l'essentiel des prestations requises par les clientèles puissent être honorées sans susciter d'aversion. Les pays en développement se prêtent avec le maximum de malléabilité à ce jeu par l'intermédiaire de leurs exigences réduites en rémunération et en bénéfices; de surcroit, l'essentiel des gains rapportés par la chaîne logistique revient aux pays porteurs des groupes d'intérêt; le transport contribue très activement, souvent dans des proportions plus élevées que dans d'autres domaines de prestation de services - hôtellerie, restauration, etc. - à alimenter le produit national brut des pays riches; par contre les sommes récupérées par la Triade pour la fourniture des autres services s'avèrent être en général plus consistantes, ce qui révèle à nouveau l'érosion accentuéedesmarges bénéficiaires des compagnies de transport et leur fragilisation progressive, source des turbulences actuelles du milieu aérien, de la réduction du nombre de compagnies viables et des restructurations en cours; la dépendance, voire les dysfonctionnements des petites firmes d'aviation nationales de bon nombre de pays en développement en sont l'une des multiples conséquences.

C'est en fonction de ce processus que le tourisme, commele développement socioéconomique en général, font partie intégrante de la démarche des gros groupes d'impulsion qui consiste à façonner un espace mondial selon le modèledela Triade. Celui-ci se fonde sur le raisonnement en vertu duquel le ratio coût/profit et les possibilités de rentabilisation des périodes creuses sont particulièrement attractifsdans les pays en développement. Selon l'équipe RECLUS dirigée par P. Brunet (Montpellier), les pays en retrait peuvent être classés grosso modo parmi les catégories suivantes:

- les périphéries dominées, genres de réservoirsd'investissement constitués e.a. par une partiedel'Amériquelatine, de l'Afrique et de l'Asic;

- les périphéries intégrées, à la fois bassins d'émission et d'accueil des investissements, tels que le Mexique, les Philippines ou le bassin méditerranéen;

- les semi-isolats, comptant sur Lurs propres forces, tels que la Chine ou l'Inde;
- les angles morts tels que le Sahara ou l'Antarctique et, plus récemment, la Russic.

Ce schéma s’applique également au tourisme, dans la mesure où, comme nous l'avons déjà précisé ci-dessus, cette filière s'inscrit entièrement et à part entière dans la structuration économique d'ensemble du globe. Il rend plus plausible encore les raisons de la subordination des pouvoirs publics et collectivités locales des pays en développement aux impératifs découlant des logiques d'emprise spatialedes groupes d'investissement touristique de la Triade: l'aménagement du territoire demeure une notion très équivoque dès lors que l'espace est hypothéqué par des partis d'aménagement inspirés du dehors. Dans son article intitulé L'enfer de la productivité (revue Espaces, 113, Paris, 1991-1992, pp. 18-23), Josquin Barré note en introduction à ses réflexions sur la ruralité en pays avancé: \&La tourismatique pourra apporter des solutions commerciales pour développer le tourisme dans le monde rural et pour rentabiliser la modernisation del hôtellerie indépendante. Lesacteurséconomiques et institutionnels du tourisme accepterontils, pour y contribuer, de revitaliser leur systèmedevaleurs, de remettre leur culture économique en question et de regrouper leurs initiatives? $\%$. Si une telle observation peut paraittre judicieuse dans un monde déjà très structuré, elle montre combien les pays fragiles sont plus que jamais exposés aux emprises variées des forces politicoéconomiques extérieures. Celles-ci sont d'autant plus à l'aise qu'elles disposent désormais de leviers de commande imperceptibles a priori à travers les dédales télématiques qui conduisent au transport aérien. Ce dernier n'est que l'élément visible et quasi-aseptisé de profondes solidarités internationales dont les appétits ne correspondent pas nécessairement - si l'on peut dire - avec les identités locales. Les acquisitions, prises de participation etautres procédés marquant la conquête territoriale du marché touristique aboutissent à l'équivoque suivante:

- d'une part, cette évolution a favorisé l'insertion des pays peu développés au restant du globe par le déploiement delignes aériennes et d'aéroports plus ou moins structurants;

- d'autre part, elle a élargi le systènemonde aux pays démunis de manière que le transport aérien soit le maillon 
d'une vaste chaîne resserrant l'étau autour d'un corps d'autant plus fragilisé que les hautes technologies l'exposent à des influences difficies sinon impossibles à contenir en attendant.

\section{Interrogations}

La réalité des mécanismes et des enjeux contraste souvent étonnamment avec l'apparence quotidienne du vécu.

L'imagerie inhérente à l'évasion continue à être soignéeet progresse dans son contenu autant que dans son efficacité. L'ouverture de petites lignes aériennes interinsulaires inaugure ou amplifie par exemple des distinctions entre les îles naturelles et les îles sauvages (c'est le cas pour la province insulaire de Nouvelle-Calédonie). En Océanie se sont esquissés l'axe du mytbe (Tahiti, Bora-Bora) et l'ave de laventure (TlesAustrales, Tuamotu, Marquises), tous deux intégrésaux circuits mondiaux dont la compagnie Thail vise d'importantes parts demarché. Tandis que Tahitiest présentée comme la nouvelle Cytbère, la NouvelleCalédonie cherche à se rattraper, des arrière-pensées politiques étant à peine dissimulées depuis que l'autonomie du territoire d'outre-mer impose des résultats en vue du référendum sur le statut final.

La question aéroportuaireestconstamment présente: à l'extrême nord de la Grande Terre, dans la Baie de la Néhoué, le Malabou Beach Hotel (trois étoiles) offre 34 bungalows et 3 suites à 400 kilomètres de Nouméa par la route qui borde la côte ouest et à 50 minutes d'avion, l'hôtel disposant d'un aérodrome privé. La Région du Nord issue des accords Matignon de 1988 envisage la construction d'un aéroport international à destination principalement touristique. Certes les flux touristiques à Tahiti ont augmenté de moitié en un an; la France, l'Allemagne, la Grande-Bretagne et l'Italie pourvoient de façon prioritaire le marché polynésien. $\mathrm{La}$ Nouvelle-Calédonie n'en est pas là; aussi les responsables du Nord caressent-ils des espoirs, au moment où la clientèle vacancière demeure faible et où l'idée de la création d'un écotourisme néo-calédonien faitsonchemin. Alors que Tahitin'a pas de compagnie aérienne internationale propre, la Nouvelle-Calédonieen possède une. Le scénario à l'horizon 2000 annonce 160 000 touristes par an. Accor et le Club
Méditerranée ont pris position sur l'archipel. Le marché japonais qui n'a fourni que 25000 touristes en 1993 semble envoyer 62000 touristes en l'an 2000 . Il s'agit cependant là d'une part minime des 23150 000 Japonais qui sillonneront le globe sous la dénomination de touristes en l'an 2000. La Chine sera toutefois à cet horizon le principal marché touristique de l'AsiePacifique, avec des dépenses équivalant à 30 milliards de francs CFP susceptibles de créer 10000 emplois. Autant de signes d'espoir et d'inquiétude face aux appétits infrastructurels actuels des responsables néo-calédoniens. La question fondamentale posée consiste à savoir sil l'éloignement du Pacifique-Sudpermettra de faire rentrer cette zone dans l'aire des grands axes touristiques del'avenir. En attendant, le Club Méditerranée dont le bâtiment de croisière Chu Med 2, le plus important navire croisiériste du globe, basé à Nouméa durant six mois par an, risque de quitter la Nouvelle-Calédonieen 1994, ne répondant pas aux exigences de rentabilité. En attendant, Nouméa enregistre 50000 touristes croisiéristes et environ 1000 plaisanciers par an. Certaines îles néocalédoniennesont une pisted'aviation pour à peine 200 habitants. En Polynésie, une île comme Bora-Bora accueille déjà 150 000 touristes par an. A Tahiti et à Mooréa, la moyenne de séjour enregistrée est d'à peine cinq journées pour respectivement quatre et deux cinquièmes des touristes. Autant d'éléments qui ne vont pas forcément dans le même sens et qui inspirent de l'incertitude.

Face à ces interrogations surgit la question des rapports entre l'ouverture touristique favorisée par les transports aériens et l'environnement physico-social des pays en développement incriminés. La surcharge touristique est d'autant plus facilement atteinte que le milieu physique est généralement fragile, d'autant plus que les emprises contemporaines sont puissantes dans leurs effets dévastateurs physiqueset sociaux. La multiplication des aéroports à vocation principalement touristique perturbe les milieux plus qu'elle ne contribue à les promouvoir, Il convient doned'en étudier une répartition judicieuse et d'en limiter les répercussions négatives. Nous assistons déjà au paradoxe suivant: la nature dite sauvage a été malmenée, voire détruite en bien des endroits par une économie sauvage qui a conduit l'homme d'un extrểne à lautre. Le transport aérien est partie intégrante de la discussion qui devrait aboutir à une réelle prise de conscience des exigences environnementales au sens global du terme, requérant l'arbitrage des pouvoirs publics etd'une autoritémondiale consensuelle en vue de maîtriser les orientations perverses de la mobilité extrềme qui caractérise les sociétés tertiaroindustrielles demeurées quelque peu plus stables des pays en yoie de développement. Le transport aérien est porté par les mêmes groupes qui sont responsables de cette carence. $f$

\section{REFERENCES BIBLOGRAPHIOUES}

CHARDON, J.-P., Ĺavion dans los Antillas, collection lles et Archipels, CRET, Universite de Bordeaux iil. jullet 1987, $177 \mathrm{p}$

Wackermann, G., Transports et dependances intemationales des pays en voie de développement, dans Travaux de I'Institut de gubographie de Roims (TIGA), 63-64, 1985, pp. 1-19.

Wackemann, G., Enclavement et mobulite en Afrique occidentale: I'exemple du Burking Faso, dans Afrique contemporaine. 140. La Documentation Française, Paris, 1986, pp. 24-39.

Wackermann, $G_{1}$ Le transport en transit des pays enclavếs d'Afrique de l'ouest et d'Afrique centrale cen collaboration avec M. Walla, Secrétaire général de la Conférence africaine des ministres des transports, Abidjanl. Projet dassis: tance, Programme des Nations-Unies, CNUCED, Doc. AAFVBVOS8/1987, Geneve.

Wackermann, G., Das Genossenschafts wesen und der Wandel der ländichen Gesellschatt in Burkind Faso, dans Fastschrift für Herrn Prof. Dr. Sick, Geographisches Institut der Universitut Freburgr i-Br., Fribourg. Allemagne, 1990.

Wackermann, G., Comportements ef vecu touristicues a la Rémion - Rétro-et prospective, dans Pers. poctives de dóveloppement tour istique de l'lle de la Róunion, Comité régional du tourisme de la Réunion, 1993.

Wackermann, G. Transport et désenclavement de r'Alrique. Conterence des Chambres de commerce françaises et africaines (Commission des transports], Chambre de commerce, Bordesux, 1993.

Wackermann. G., Tourisme ot transport, collection Mobilité spatiale, Editions SEDES, Paris, 1990. $279 \mathrm{p}$. 